\title{
Modulation effect of the atmosphere in a pyramid wave-front sensor
}

\author{
Joana B. Costa
}

\begin{abstract}
The pyramid wave-front sensor in its original form works with a mechanical modulation that adapts the linear range of the sensor to seeing and sensing conditions. For adaptive optics systems working in an astronomical context, the way in which the aberrations produced by the atmospheric turbulence, which are not seen by the sensor owing to its limited temporal bandwidth, act as modulators is shown. These aberrations have the same effect of increasing the linear range and localizing the measurement as does mechanical modulation. The effect of residual wave-front aberrations is estimated for some example conditions of telescope diameter, system bandwidth, wind velocity, and Fried parameter. (C) 2005 Optical Society of America

OCIS codes: $\quad 010.1080,010.7350$.
\end{abstract}

\section{Introduction}

Since the pyramid wave-front sensor (PWS) was proposed, ${ }^{1}$ it has been studied in the laboratory ${ }^{2}$ and has been successfully operated with a telescope, implemented in the adaptive optics module of the Telescopio Nazionale Galileo ${ }^{3}$ to measure the aberrations produced by atmospheric turbulence that degrade astronomical images. In its original form the PWS works with mechanical modulation that ensures a certain dynamic range, such that the signal does not saturate and quantitative statements about the wave-front's derivative are possible. One can achieve this modulation in various ways, e.g. with a tip-tilt mirror located in the pupil plane, by moving the pyramid, or with a light-diffusing plate. ${ }^{4}$ It has been shown through numerical simulations that there is always an ideal amplitude for this modulation that maximizes the sensitivity in conditions of low light levels. ${ }^{5}$ An interesting property of this type of sensor is the gain in sensitivity that is expected in comparison to a Shack-Hartmann sensor, ${ }^{6,7}$ when the PWS is operating in closed loop and is not modulated. However, one has to have in mind that, if the aberrations exceed the linear range of the sensor, which is quite small in the unmodulated case, the signal gets satu-

J. Costa (costa@mpia.de) is with the Max-Planck-Institute for Astronomy, Koenigstuhl 17, Heidelberg, Germany 69117.

Received 16 April 2004; revised manuscript received 21 September 2004; accepted 5 October 2004.

0003-6935/05/010060-07\$15.00/0

(C) 2005 Optical Society of America rated. It has been pointed out that residual aberrations from atmospheric turbulence, which have the effect of enlarging the spot size on the pyramid, can themselves act as modulators. ${ }^{8}$ This is an interesting idea, because it would greatly simplify the optical and mechanical design of adaptive optic systems that use PWSs as well as contribute to the interpretation of the measurements given by a PWS. In this paper it is shown analytically how the aberrations produced by atmospheric turbulence, which are not seen by the sensor because of its limited temporal bandwidth, have the same effect as modulation and how this effect can be quantified.

In Section 2 the effects of modulation on the signals given by the sensor are described. In Section 3 a model is developed to describe how residual aberrations, not sensed by the PWS owing to its temporal bandwidth, can act as natural modulators. Then in Section 4 a quantitative analysis is performed that gives some examples of the size of this effect.

\section{Modulation and Its Effects on the Sensor Signal}

To understand the effect of modulation on the PWS it is enough to study the simpler case of modulation on a knife-edge sensor. For a detailed derivation of the equations in this section the reader is referred to the research of Feeney. ${ }^{9} \mathrm{~A}$ brief résumé is given in Appendix A. We start with the expression for the signal in the pupil of the knife-edge wave-front sensor, where the knife-edge is located along the $y$ axis, and we measure only signals in the $x$-axis direction $\left(S_{x}\right)$ (Refs. 9-11): 
$S_{x}(x, y)=\frac{1}{\pi} \int_{-B(y)}^{B(y)} \mathrm{d} x^{\prime}(\mathrm{p} v) \frac{\sin \left[\phi\left(x^{\prime}, y\right)-\phi(x, y)\right]}{\left(x^{\prime}-x\right)}$,

where $\mathrm{p} v$ means the principal-value distribution. The integral is performed along a chord in the pupil, perpendicular to the knife-edge, which contains point $(x, y)$ where the signal is being computed. As represented in Fig. 1, $\pm B(y)$ are the edge points of the chord and $\phi(x, y)$ is the wave-front phase at point $(x, y)$. In the integral the contribution of the sine term is weighted by the distance between the integration point $\left(x^{\prime}, y\right)$ and the point where the signal is being measured $(x, y)$.

With a tilt term oscillating perpendicularly to the knife-edge, the expression for the signal extends to ${ }^{9}$

$$
\begin{aligned}
S_{x}(x, y)= & \frac{1}{\pi} \int_{-B(y)}^{B(y)} \mathrm{d} x^{\prime}(\mathrm{p} v) \frac{\sin \left[\phi\left(x^{\prime}, y\right)-\phi(x, y)\right]}{\left(x^{\prime}-x\right)} \\
& \times \frac{\Delta t \frac{\sin \left[a_{0}\left(x^{\prime}-x\right)\right]}{a_{0}\left(x^{\prime}-x\right)} .}{\text { modulation }} .
\end{aligned}
$$

The signal has been averaged over one modulation period $\Delta t$, and $a_{0}$ is the modulation amplitude. As $\sin x / x \rightarrow 1$, for small $x$ the term added to the integral reduces the contribution of the more distant pupil points to the signal at $(x, y)$, linearizing the signal such that, for sufficiently large modulation amplitude, the signal measured will correspond to the local derivative of the wave front.

\section{Model to Describe the Natural Modulation}

One can decompose the phase in the pupil $\phi(x, y ; t)$ into polynomial terms:

$$
\phi(x, y ; t)=\sum_{n} c_{n}(t) A_{n}(x, y)
$$

where $A_{n}(x, y)$ is a complete set of functions that are orthogonal over a unit circle and $c_{n}(t)$ are the timevarying coefficients. We assume that the functions $A_{n}(x, y)$ have a diagonal covariance matrix (as do Karhùnen-Loeve polynomials). For every $c_{n}$ there is a Gaussian distribution with a variance $\sigma_{n}$ for each $c_{n}$, which gives the probability of finding a certain value:

$$
P\left(c_{n}\right)=\frac{1}{\sigma_{n} \sqrt{2 \pi}} \exp \left(-\frac{c_{n}{ }^{2}}{2 \sigma_{n}}\right) .
$$

We know that the measured signal is obtained from integration of the signal during time $T$. So we can separate $\phi(x, y ; t)$ into two parts: one that changes little on time scales smaller than or equal to $T$ and therefore for practical purposes is static and can be measured and another that changes in a statistical way on such a time scale (and thus cannot be measured).

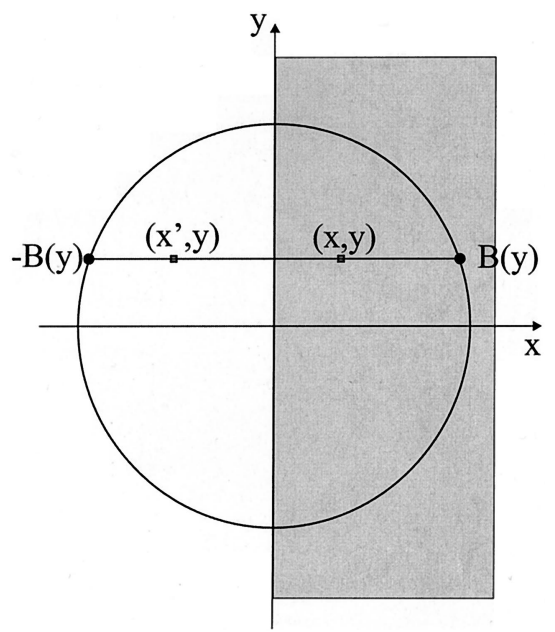

Fig. 1. Signal computation for the knife-edge wave-front sensor

Let us therefore separate $\phi(x, y ; t)$ into two parts:

$$
\phi(x, y ; t)=\phi_{s}(x, y)+\sum_{j} c_{j}(t) A_{j}(x, y) .
$$

Here $\phi_{s}(x, y)$ is the part that can be measured (because it changes little during $T$ ). The second term is the part of the phase that cannot be measured with our sensor because of temporal limits.

For the mean value of the signal over one measurement cycle we get

$$
\left\langle S_{x}(x, y)\right\rangle=\frac{1}{T} \int_{0}^{T} \mathrm{~d} t S_{x}(x, y ; t) .
$$

Substituting Eqs. (1) and Eq. (5) into Eq. (6) and replacing the integral over time with an integral over all possible configurations of $c_{j}$ multiplied by their probability, we get

$$
\begin{aligned}
\left\langle S_{x}(x, y)\right\rangle= & \frac{1}{C} \int_{+\infty}^{-\infty} \prod_{j} \mathrm{~d} c_{j} \frac{1}{\pi} \int_{-B(y)}^{B(y)} \mathrm{d} x^{\prime} \\
& \times(\mathrm{p} v) \frac{\sin \left[\phi_{s}\left(x^{\prime}, y\right)-\phi_{s}(x, y)\right]}{x^{\prime}-x} \\
& \times \cos \left\{\sum_{j} c_{j}\left[A_{j}\left(x^{\prime}, y\right)-A_{j}(x, y)\right]\right\} \\
& \times \exp \left(-\sum_{j} \frac{c_{j}^{2}}{2 \sigma_{j}}\right) .
\end{aligned}
$$

Equation (7) can be rewritten as

$$
\begin{aligned}
\left\langle S_{x}(x, y)\right\rangle= & \frac{1}{\pi} \int_{-B(y)}^{B(y)} \mathrm{d} x^{\prime}(\mathrm{p} v) \frac{\sin \left[\phi_{s}\left(x^{\prime}, y\right)-\phi_{s}(x, y)\right]}{x^{\prime}-x} \\
& \times M\left(x^{\prime}, x, y\right),
\end{aligned}
$$

where modulation $M\left(x^{\prime}, x, y\right)$ is 


$$
\begin{aligned}
M\left(x^{\prime}, x, y\right) \equiv & \frac{\Pi_{j}}{C} \int_{-\infty}^{+\infty} \mathrm{d} c_{j} \exp \left\{i c _ { j } \left[A_{j}\left(x^{\prime}, y\right)\right.\right. \\
& \left.\left.-A_{j}(x, y)\right]\right\} \exp \left(-\frac{c_{j}^{2}}{2 \sigma_{j}}\right) .
\end{aligned}
$$

It should be noted that Eqs. (8) and (9) are similar to Eq. (2).

With $\int_{-\infty}^{+\infty} \mathrm{d} c \exp \left[-\left(c^{2} / 2 \sigma\right)+i c A\right] \propto \exp$ $\left[-\left(\sigma A^{2} / 2\right)\right]$, one gets

$M\left(x^{\prime}, x, y\right)=\exp \left\{-\sum_{j} \frac{\sigma_{j}}{2}\left[A_{j}\left(x^{\prime}, y\right)-A_{j}(x, y)\right]^{2}\right\}$.

Clearly, the modulation function satisfies $M(x, x, y)=1$ and $M\left(x^{\prime}, x, y\right) \leq 1$ and, if $\sigma_{j} \neq 0$ for several $j, M$ has the effect of cutting off the integral for large $x^{\prime}-x$, linearizing $\sin \left[\phi_{s}\left(x^{\prime}, y\right)-\phi_{s}(x, y)\right]$ in the integral, such that

$$
\left\langle S_{x}(x, y)\right\rangle \rightarrow C \frac{\mathrm{d} \phi_{s}(x, y)}{\mathrm{d} x}
$$

for a constant $C$. This is exactly the same as the effect of mechanical modulation.

\section{Quantitative Estimation of the Atmospheric Modulation Effect}

To make a quantitative estimate of the shape of the modulation function defined in Section 3 we calculate the temporal power spectrum of each Zernike mode, ${ }^{12}$ where $v$ is the temporal frequency and $v / V$ is the spatial frequency, $f_{x}$ :

$$
\begin{aligned}
w_{Z_{j}}(v)= & 0.033 \frac{C_{N}^{2} d h}{V}(2 \pi)^{-2 / 3}\left(\frac{2 \pi}{\lambda}\right)^{2} \\
& \times \int_{-\infty}^{+\infty} \mathrm{d} f_{y}\left|\tilde{Z}_{j}\left(\frac{v}{V}, f_{y}\right)\right|^{2}\left[\left(\frac{v}{V}\right)^{2}+f_{y}^{2}\right]^{-11 / 6},
\end{aligned}
$$

where $\tilde{Z}\left(f_{x}, f_{y}\right)$ is the Fourier transform of the $j$ th Zernike polynomial $Z_{j}(x, y),\left(f_{x}, f_{y}\right)$ is spatial frequency vector $f$, and $n$ and $m$ are the radial degree and the azimuthal frequency, respectively:

$$
\begin{aligned}
\left|\tilde{Z}_{j}\left(f_{x}, f_{y}\right)\right|= & (n+1)^{1 / 2} \frac{2\left|J_{n+1}(\pi D f)\right|}{\pi D f} \\
& \times\left\{\begin{array}{cl}
\sqrt{2}|\cos (m \theta)| & m \neq 0 \\
\sqrt{2}|\sin (m \theta)| & m \neq 0 \\
1 & m=0
\end{array}\right.
\end{aligned}
$$

Wind velocity $V$ is taken along the $x$ direction (to generalize to any wind direction, one just has to rotate the axes such that the new $f_{x}$ is parallel to the wind direction). The Taylor hypothesis of frozen tur-

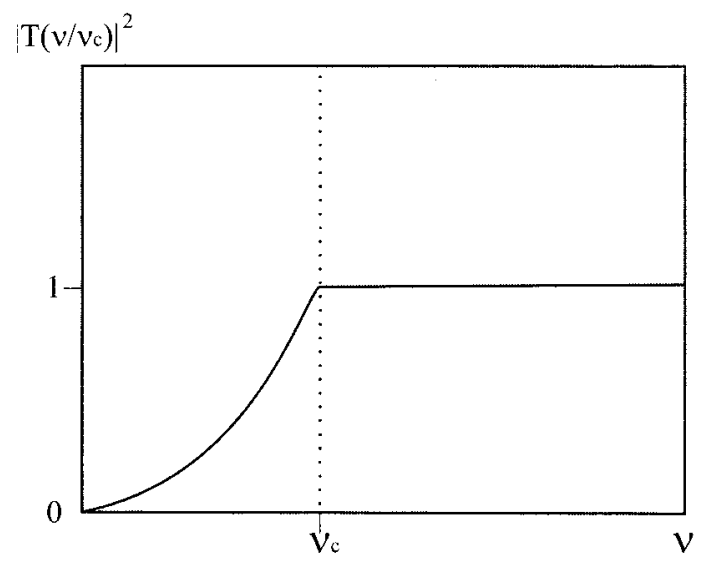

Fig. 2. Square modulus of the error transfer function for a system with bandwidth $\nu_{c}$.

bulence is assumed. The index structure coefficient is $C_{N}{ }^{2}$, the layer thickness is $d h$, and the wavelength is $\lambda$.

To get the residual variance, $\sigma_{j}$, one has to weigh Eq. (12) with the error transfer function square modulus $|T(v)|^{2}$ and integrate it:

$$
\sigma_{j}=\int_{-\infty}^{+\infty}|T(v)|^{2} w_{Z_{j}}(\nu) \mathrm{d} \nu,
$$

where we take

$$
\left|T\left(\frac{v}{v_{c}}\right)\right|^{2}=\left(\frac{v}{v_{c}}\right)^{2} \theta\left[1-\left(\frac{v}{v_{c}}\right)^{2}\right]+\theta\left[\left(\frac{v}{v_{c}}\right)^{2}-1\right] .
$$

Here $v_{c}$ is the system's bandwidth and $\theta$ represents the step function, which is 0 for negative input values and 1 elsewhere. Function $T$ is represented in Fig. 2.

This means that there will be a residual contribution to the error from frequencies $v<v_{c}$. Frequencies with $v>v_{c}$ contribute with weight 1 . In practice what I did was to calculate

$$
\sigma_{j}=\frac{\int_{-\infty}^{+\infty}|T(v)|^{2} w_{Z_{j}}(v) \mathrm{d} v}{\int_{-\infty}^{+\infty} w_{Z_{j}}(v) \mathrm{d} v}\left(\Delta_{j}-\Delta_{j+1}\right),
$$

with $\Delta_{j}$ given by the Zernike-Kolmogorov residual errors $^{13,14}$ :

$$
\Delta_{j} \approx 0.2944 j^{-\sqrt{3} / 2}\left(\frac{D}{r_{0}}\right)^{5 / 3}
$$

Here $D$ is the telescope diameter and $r_{0}$ is the Fried parameter. The residual variance $\sigma_{j}$ can be written as 


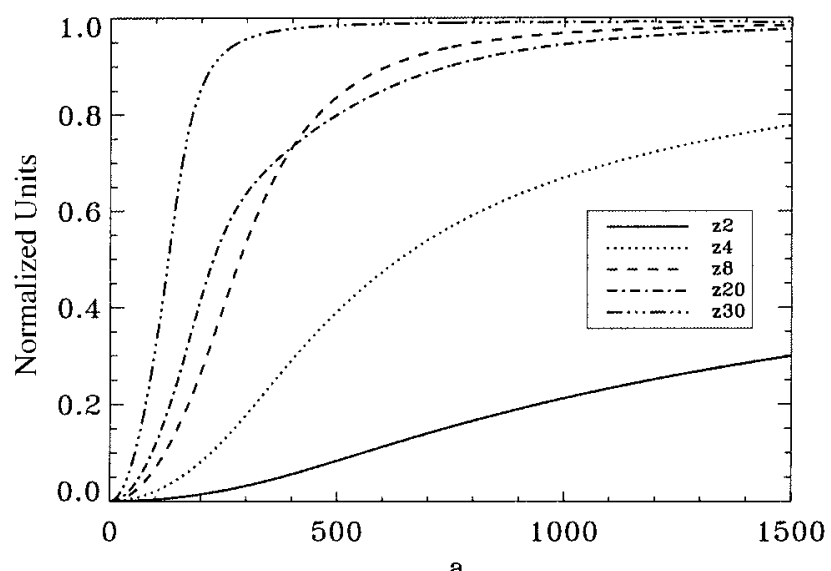

Fig. 3. Normalized residual variance $\sigma_{j} /\left(\Delta_{j}-\Delta_{j+1}\right)$ for some Zernike polynomials as a function of $a$, the ratio of the wind speed over the system's bandwidth multiplied by the telescope's diameter $\left[a=V /\left(\nu_{c} D \pi\right)\right]$.

Eq. (16) because $\int_{-\infty}^{+\infty} w_{Z_{j}}(v) \mathrm{d} v=\left(\Delta_{j}-\Delta_{j+1}\right)$, which allows all the constants outside the integrals to be dropped. For the derivation and numerical integration of the equations refer to Appendix B.
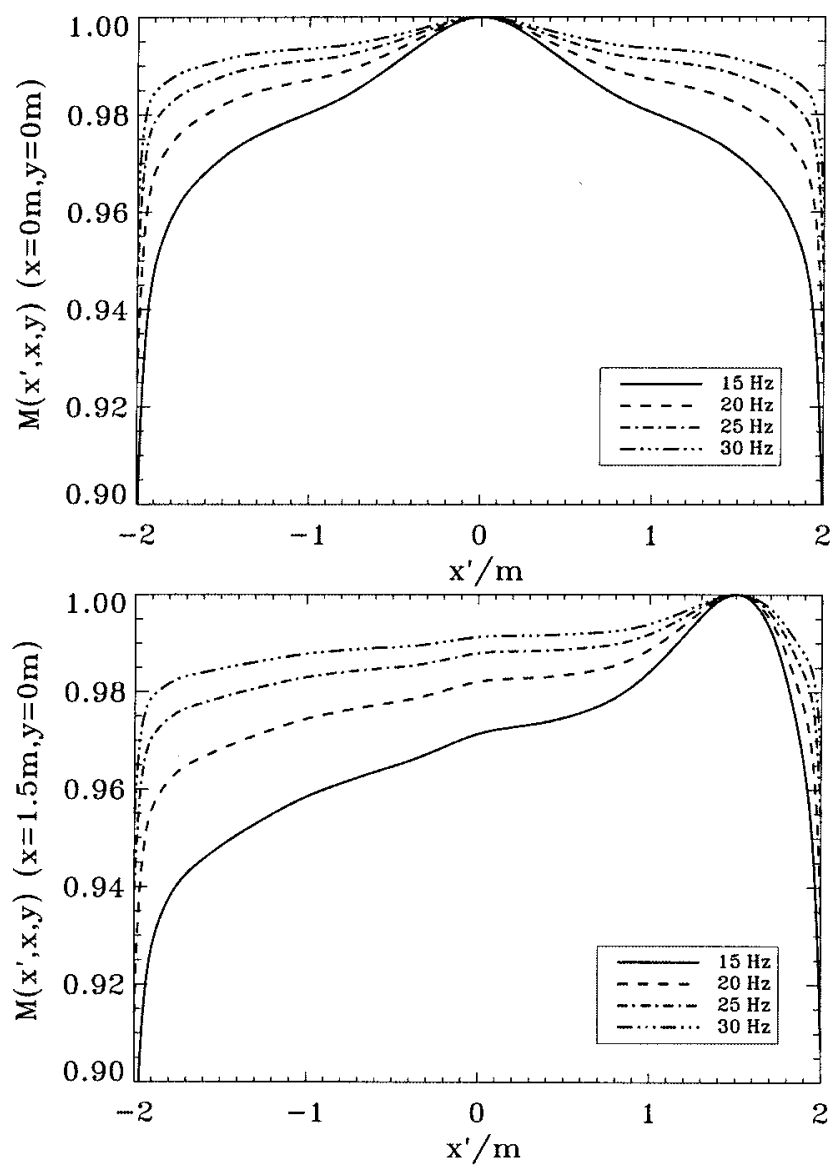

Fig. 4. Modulation function across the telescope aperture [top, in the pupil center and bottom, at point $(x=1.5 \mathrm{~m}, y=0 \mathrm{~m})$ ] for several system bandwidths $v_{c}$, for a telescope diameter of $4 \mathrm{~m}$, a wind velocity of $30 \mathrm{~m} / \mathrm{s}$, and an $r_{0}$ of $1 \mathrm{~m}$.

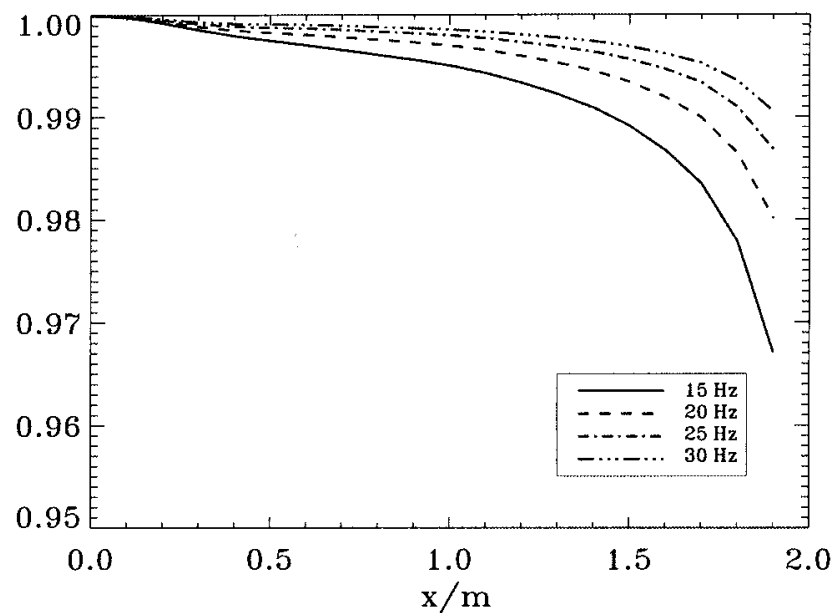

Fig. 5. Integrated modulation function across the telescope aperture for several points along the $y=0$ chord, normalized with the integral at $(x=0 m, y=0 \mathrm{~m})$ for several system bandwidths $v_{c}$, for a telescope diameter of $4 \mathrm{~m}$, a wind velocity of $30 \mathrm{~m} / \mathrm{s}$, and an $r_{0}$ of $1 \mathrm{~m}$.

The modulation function obtained depends on a variable, which we define as $a=V /\left(\nu_{c} D \pi\right)$, and on $D r_{0}{ }^{5}$ (see Appendix C). Its effect will increase with higher wind velocity $V$ and decrease with bigger telescope diameters $(D)$, higher $r_{0}$ (better seeing), and higher system bandwidth $v_{c}$. This is illustrated in Fig. 3 , where the normalized residual variance for several Zernike modes is plotted as a function of $a$. The modulation function has also been plotted for certain conditions, and a map has been made over the pupil for two pupil positions (see Fig. 4, for example).

The modulation function has been integrated across the pupil chord for the plots presented, excluding the point where it has the value 1 , and normalized with the integral of the modulation function at the pupil center; the results are shown in Fig. 5. The value obtained gives an estimate of the suppression of the contribution of points on the same chord to the signal. The modulation effect does not change more than 1-2\% over the pupil, at least under the conditions simulated. It shows a slightly greater modulation effect at the edges of the pupil. Further analysis to determine whether this could have a physical meaning or could be due to numerical approximations would be useful but is beyond the scope of this paper.

Here I have used Zernike modes, neglecting the nonzero terms in the covariance matrix. The numerical analysis is done in the same way with KarhùnenLoeve polynomials, but I expect no significant difference in the magnitude of the modulation obtained.

For comparison with the mechanical modulation, the atmospheric modulation and the effect of tilt modulation with amplitude in $\lambda / D$ units has been plotted in Fig. 6. The size of the diffraction-limited spot on the pyramid tip corresponds to $1 \lambda / D$. To move the complete spot over all the pyramid sides the smallest amplitude is $0.5 \lambda / D$. In the conditions simulated, the atmospheric modulation effect is comparable to a mechanical modulation of $0.2 \lambda / D$. 


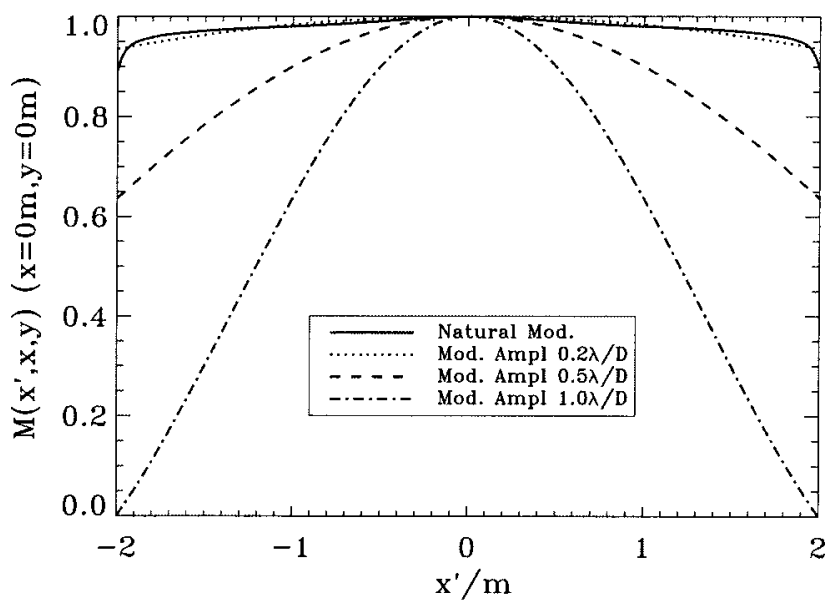

Fig. 6. Modulation function for a telescope with diameter of $4 \mathrm{~m}$ with a wind velocity of $30 \mathrm{~m} / \mathrm{s}, r_{0}=1 \mathrm{~m}$, and a system bandwidth of $15 \mathrm{~Hz}$ (for $x=0, y=0$ ) (solid curve). For comparison we have also plotted the mechanical modulation function for three modulation amplitudes.

\section{Conclusions}

It has been shown that the residual aberrations of the atmospheric turbulence not seen by the sensor because of the system's limited temporal bandwidth can themselves act as modulators, contributing to the linearization of the signal. This effect can be numerically estimated for each condition of telescope diameter, atmospheric condition (wind velocity and $r_{0}$ ), and system bandwidth. A comparison of this so-called natural modulation with the mechanical modulation of the PWS signal shows that in certain conditions the two may have comparable effects.

It is known that modulation permits better localization of the signal measured, for which the PWS approaches a local slope sensor. This will be naturally, and unavoidably, increased by atmospheric turbulence, with its potentially good effects, such as increased linear range of the sensor, and bad effects, one of which is loss of delocalized information. The ability to use this information is actually one of the potential advantages of the PWS in the nonmodulated regime.

Here we estimate the effect of only one atmospheric layer, whereas the estimation can in the same way be generalized to more layers, which will of course increase the modulation effect.

It would be interesting to investigate further where the point lies at which the nonlinearities do not allow the adaptive optics loop to close, which is equivalent to the amount of modulation is needed in each case. For every case, when the optimal modulation amplitude is estimated the natural modulation should also be taken into account. It may be enough to close the loop and achieve the performance needed, especially when the common path aberrations that enlarge the spot on the pyramid and therefore facilitate a greater linear range are small.
A further analysis of the effect of modes that were not seen because of the spatial sampling characteristics of the sensor would be interesting here.

\section{Appendix A. Expression for the Sensor Signal}

Starting from the expression of Linfoot ${ }^{11}$ and taking image inversion into account ${ }^{10}$ allow the complex amplitude of the electromagnetic field in the image plane after diffraction at a knife-edge along the $y$ axis to be written as $^{9}$

$$
u_{i}^{\mp}(x, y)=\frac{1}{2} u_{0}(x, y) \pm \frac{i}{2 \pi} \int_{-B(y)}^{B(y)} \mathrm{d} x^{\prime}(\mathrm{p} v) \frac{u_{0}\left(x^{\prime}, y\right)}{\left(x^{\prime}-x\right)}
$$

where $u_{0}\left(x^{\prime}, y\right)$ is the complex electromagnetic field amplitude in the object plane and $\pm B(y)$ are the edge points of a chord perpendicular to the knife-edge at coordinate $y . \pm$ are two complementary positions that have the negative and the positive sides covered. As the observable is the absolute square of the complex amplitude, it can be calculated with

$$
\begin{aligned}
\left|u_{i}^{+}(x, y)\right|^{2}= & {\left[\frac{1}{2} u_{0}(x, y)-\frac{i}{2 \pi} \int_{-B(y)}^{B(y)} \mathrm{d} x^{\prime}(\mathrm{p} v) \frac{u_{0}\left(x^{\prime}, y\right)}{\left(x^{\prime}-x\right)}\right] } \\
& \times\left[\frac{1}{2} u_{0}^{*}(x, y)+\frac{i}{2 \pi} \int_{-B(y)}^{B(y)} \mathrm{d} x^{\prime}\right. \\
& \left.\times(\mathrm{p} v) \frac{u_{0}^{*}\left(x^{\prime}, y\right)}{\left(x^{\prime}-x\right)}\right],
\end{aligned}
$$

and similarly for $\left|u_{i}{ }^{-}(x, y)\right|^{2}$. The signals in the $x$ direction can be calculated as

$$
S_{x}(x, y)=\frac{\left|u_{i}^{-}\right|^{2}-\left|u_{i}^{+}\right|^{2}}{\left|u_{0}\right|^{2}} .
$$

Substituting the previous expressions (A2) for $\left|u_{i}{ }^{ \pm}\right|^{2}$ and $u_{0}(x, y)=E \exp [-i \phi(x, y)]$ into (A3), one gets

$$
\begin{aligned}
S_{x}= & \frac{2 i}{4 \pi}\left[u_{0}^{*} \int_{-B(y)}^{B(y)} \mathrm{d} x^{\prime}(\mathrm{p} v) \frac{u_{0}\left(x^{\prime}, y\right)}{\left(x^{\prime}-x\right)}\right. \\
& \left.-u_{0} \int_{-B(y)}^{B(y)} \mathrm{d} x^{\prime}(\mathrm{p} v) \frac{u_{0}^{*}\left(x^{\prime}, y\right)}{\left(x^{\prime}-x\right)}\right],
\end{aligned}
$$

and, after some simplifications,

$$
S_{x}(x, y)=\frac{1}{\pi} \int_{-B(y)}^{B(y)} \mathrm{d} x^{\prime}(\mathrm{p} v) \frac{\sin \left[\phi\left(x^{\prime}, y\right)-\phi(x, y)\right]}{\left(x^{\prime}-x\right)}
$$

which corresponds to Eq. (1). 
In the simplified knife-edge case the modulation can be reduced to a linear movement of the beam perpendicular to the edge, where the phase is increased by an oscillating tilt term with amplitude $a_{0}$ and period in time $\Delta t$ :

$$
\widetilde{\phi}(x, y ; t)=\phi(x, y)+a_{0} \frac{2 t}{\Delta t} x
$$

for time $t$. Substitution into Eq. (A5) and integration over one period yield Eq. (2).

\section{Appendix B. Integration of the Power Spectrum}

A method of getting expressions that are easily integrable and depend on only one parameter is described here. First we rewrite the power spectrum for each mode $Z_{n}$ :

$$
\begin{aligned}
w_{Z_{j}}(\nu)= & 4(n+1) C_{1} \int_{-\infty}^{+\infty} \mathrm{d} f_{y} \frac{\left|J_{n+1}(\pi D f)\right|^{2}}{\pi D f}\left[\left(\frac{\nu}{V}\right)^{2}\right. \\
& \left.+f_{y}^{2}\right]^{-11 / 6}\left\{\begin{array}{cl}
2 \cos ^{2}(m \theta) & m \neq 0 \\
2 \sin ^{2}(m \theta) & m \neq 0 \\
1 & m=0
\end{array}\right.
\end{aligned}
$$

where $f=\left(f_{x}^{2}+f_{y}^{2}\right)^{1 / 2}, f_{x}=f \cos \theta=v / V$, and $f_{y}$ $=f \sin \theta$.

Performing the variable change $\mu=V f_{y}$ yields

$$
\begin{aligned}
w_{Z_{j}}(v)= & \frac{4(n+1) C_{1} V^{14 / 3}}{\pi^{2} D^{2}} \int_{-\infty}^{+\infty} \mathrm{d} \mu \\
C_{2} & \\
& \times \frac{\left|J_{n+1}\left[(\pi D / V)\left(v^{2}+\mu^{2}\right)^{1 / 2}\right]\right|^{2}}{\left(v^{2}+\mu^{2}\right)^{17 / 6}}
\end{aligned}
$$

$$
\times\left\{\begin{array}{cl}
2 \cos ^{2}\left\{m \arcsin \left[\mu /\left(v^{2}+\mu^{2}\right)^{1 / 2}\right]\right\} & m \neq 0 \\
2 \sin ^{2}\left\{m \arcsin \left[\mu /\left(v^{2}+\mu^{2}\right)^{1 / 2}\right]\right\} & m \neq 0 \\
1 & m=0
\end{array} .\right.
$$

Now we integrate the power spectrum for each mode $n$, changing to polar coordinates $(r, \theta)$ with $r^{2}$ $=v^{2}+\mu^{2}$ :

$$
\begin{aligned}
\int_{-\infty}^{+\infty} \mathrm{d} \nu w_{Z_{j}}(v)= & C_{2} \int_{0}^{2 \pi} \mathrm{d} \theta \int_{0}^{+\infty} \mathrm{d} r \frac{\left|J_{n+1}(\pi D r / V)\right|^{2}}{r^{14 / 3}} \\
& \times\left\{\begin{array}{cc}
2 \cos ^{2}(m \theta) & m \neq 0 \\
2 \sin ^{2}(m \theta) & m \neq 0 \\
1 & m=0
\end{array}\right. \\
= & 2 \pi C_{2} \int_{0}^{+\infty} \mathrm{d} r \frac{\left|J_{n+1}(\pi D r / V)\right|^{2}}{r^{14 / 3}}
\end{aligned}
$$

Changing variables again, $\rho=(\pi D r) / V$, yields

$$
\int_{-\infty}^{+\infty} \mathrm{d} \nu w_{Z_{j}}(\nu)=2 \pi C_{2}\left(\frac{V}{\pi \mathrm{D}}\right)^{-11 / 3} \int_{0}^{+\infty} \mathrm{d} \rho \frac{\left|J_{n+1}(\rho)\right|^{2}}{\rho^{14 / 3}} .
$$

Finally, I show how to integrate the power spectrum weighted with the error transfer function (in polar coordinates as before), defining $a=V /\left(\pi D v_{c}\right)$ :

$$
\begin{aligned}
\int_{-\infty}^{+\infty} \mathrm{d} \nu\left|T\left(\frac{v}{v_{c}}\right)\right|^{2} w_{Z_{j}}(\nu)=C_{2}\left(\frac{\pi D}{V}\right)^{11 / 3} \\
\times \int_{0}^{2 \pi} \mathrm{d} \theta \int_{0}^{+\infty} \mathrm{d} \rho|T(a \rho \cos \theta)|^{2} \frac{\left|J_{n+1}(\rho)\right|^{2}}{\rho^{14 / 3}} \\
\times\left\{\begin{array}{cc}
2 \cos ^{2}(m \theta) & m \neq 0 \\
2 \sin ^{2}(m \theta) & m \neq 0 \\
1 & m=0 .
\end{array}\right.
\end{aligned}
$$

Substituting $T$ from Eq. (15) into Eq. (B6) allows the integration to be performed numerically, and we see that the result, when it is normalized with Eq. (B5), depends only on $a$ (and on $j$ ).

\section{Appendix C. Dependencies of the Modulation Function}

Here we rewrite Eq. (10), introducing the normalization with the telescope diameter $D$ :

$$
M\left(x^{\prime}, x, y\right)=\exp \left\{-\sum_{j} \frac{2 \sigma_{j}}{D^{2}}\left[P_{j}\left(x^{\prime}, y\right)-P_{j}(x, y)\right]^{2}\right\} .
$$

Identifying the outcome of Eq. (B6) as $f_{j}(a)$ leads to

$$
\sigma_{j} \propto f_{j}(a) j^{-\sqrt{3} / 2}\left(D / r_{0}\right)^{5 / 3} .
$$

We can rewrite the exponential term of the modulation function:

$$
\begin{aligned}
M\left(x^{\prime}, x, y\right) \propto[ & \left.\exp \left(-\frac{1}{D^{2}}\right)\left(\frac{D}{r_{0}}\right)^{5 / 3}\right]\left(\operatorname { e x p } \left\{-\sum_{j} f_{j}(a) j^{-\sqrt{3} / 2}\right.\right. \\
& \left.\left.\times\left[P_{j}\left(x^{\prime}, y\right)-P_{j}(x, y)\right]^{2}\right\}\right) .
\end{aligned}
$$

From the first exponential we can see that $M\left(x^{\prime}, x, y\right)$ depends on $D r_{0}{ }^{5}$. The second exponential shows the dependence on $a$.

I thank Filipe Paccetti, Wolfgang Gaessler, Roberto Ragazzoni, and Markus Feldt for numerous fruitful and clarifying discussions, for useful comments, and for careful reading of the manuscript. 


\section{References}

1. R. Ragazzoni, "Pupil plane wavefront sensing with an oscillating prism," J. Mod. Opt. 43, 289-293 (1996).

2. S. Esposito, O. Feeney, and A. Riccardi, "Laboratory test of a pyramid wavefront sensor," in Adaptive Optical System Technology, P. L. Wizinowich, ed., Proc. SPIE 4007, 416-422 (2000).

3. A. Ghedina, M. Cecconi, R. Ragazzoni, J. Farinato, A. Baruffolo, G. Crimi, E. Diolati, S. Esposito, L. Fini, M. Ghigo, E. Marchetti, T. Niero, and A. Puglisi, "On sky test of pyramid wavefront sensor," in Adaptive Optical System Technologies II, P. L. Wizinowich and D. Bonaccini, eds., Proc. SPIE 4839, 869-877 (2003).

4. R. Ragazzoni, E. Diolati, and E. Viard, "A pyramid wavefront sensor with no dynamic modulation," Opt. Commun. 208, 51-60 (2002).

5. S. Esposito and A. Riccardi, "Pyramid wavefront sensor behaviour in partial correction adaptive optic systems," Astron. Astrophys. 369, L9-L12 (2001).

6. R. Ragazzoni and J. Farinato, "Sensitivity of a pyramidic wavefront sensor in closed loop adaptive optics," Astron. Astrophys. 305, L23-L26 (1999).

7. C. Vérinaud, "On the nature of the measurements provided by a pyramid wave-front sensor," Opt. Commun. 233, 27-38 (2004).

8. J. B. Costa, R. Ragazzoni, A. Ghedina, M. Carbillet, C. Verinaud, M. Feldt, S. Esposito, E. Puga, and J. Farinato, "Is there need of any modulation in the pyramid wavefront sensor?" in Adaptive Optical System Technologies II, P. L. Wizinowich and D. Bonaccini, eds., Proc. SPIE 4839, 288-298 (2003).

9. O. Feeney, "Theory and laboratory characterisation of novel wavefront sensor for adaptive optics systems," Ph.D. dissertation (National University of Ireland, Galway, Ireland, 2001).

10. R. G. Wilson, "Wavefront-error evaluation by mathematical analysis of experimental Foucault-test data," Appl. Opt. 14, 2286-2297 (1975).

11. E. H. Linfoot, "On the theory of the zonal Foucault test," Mon. Not. R. Astron. Soc. 108, 428-445 (1948).

12. J. M. Conan, G. Rousset, and P.-Y. Madec, "Wave-front temporal spectra in high-resolution imaging through turbulence," J. Opt. Soc. Am. A 12, 1559-1570 (1995).

13. D. L. Fried, "Statistics of a geometric representation of wavefront distortion," J. Opt. Soc. Am. A 55, 1427-1435 (1965).

14. R. J. Noll, "Zernike polynomials and atmospheric turbulence," J. Opt. Soc. Am. A 66, 207-211 (1976). fault fault 